\title{
Quaderni
}

QUADERNI Communication, technologies, pouvoir

\section{Un dispositif sociotechnique à la loupe :le développement de la vidéosurveillance dans trois villes françaises}

Anne-Cécile Douillet, Laurence Dumoulin et Séverine Germain

\section{(2) OpenEdition \\ Journals}

Édition électronique

URL : http://journals.openedition.org/quaderni/369

DOI : 10.4000/quaderni.369

ISSN : 2105-2956

Éditeur

Les éditions de la Maison des sciences de l'Homme

\section{Édition imprimée}

Date de publication : 15 janvier 2011

Pagination : 105-120

Référence électronique

Anne-Cécile Douillet, Laurence Dumoulin et Séverine Germain, « Un dispositif sociotechnique à la loupe :le développement de la vidéosurveillance dans trois villes françaises », Quaderni [En ligne], 74 | Hiver 2010-2011, mis en ligne le 05 janvier 2013, consulté le 01 mai 2019. URL : http:// journals.openedition.org/quaderni/369; DOI : 10.4000/quaderni.369 


\section{Technique}

\section{un dispositif sociotechnique à la loupe :}

le développement de la vidéosurveillance dans trois villes françaises ${ }^{1}$

\section{Anne-Cécile Douillet}

Université de Franche-Comté

\section{Laurence Dumoulin} CNRS-ISP Cachan

\section{Séverine Germain}

Université de Rouen
Depuis 2007, le gouvernement français a plusieurs fois annoncé le triplement du nombre de caméras de vidéosurveillance, estimé alors à $340000^{2}$, pour atteindre le chiffre symbolique du million ${ }^{3}$. Pour mesurer la portée d'une telle promotion de la vidéosurveillance, il peut être utile de revenir sur les dynamiques qui alimentent le développement de cet outil d'action publique, en particulier la vidéosurveillance de voie publique ${ }^{4}$.

La vidéosurveillance peut être appréhendée comme une innovation technologique - associant de la vidéo à des systèmes de transmission de données - mobilisée en réponse aux «problèmes d'insécurité urbaine». Cependant, plus qu'une simple technologie, c'est un ensemble d'objets dont des caméras et des moniteurs -, de normes techniques ou juridiques, de représentations, de pratiques professionnelles, d'intérêts et d'individus qui contribuent à effectuer une surveillance à distance. S'intéresser au développement de la vidéosurveillance, c'est donc analyser la montée en puissance d'un dispositif sociotechnique ${ }^{5}$. Si nous utilisons ce terme, c'est pour rappeler d'une part que l'objet est inséparable des acteurs qui le construisent, le portent et se l'approprient et, d'autre part, que l'objet est à la fois le produit de concepteurs - et de l'image qu'ils se font des utilisateurs et des 'bonnes' utilisations - et des appropriations que les opérateurs déploient en situation. Ce qu'il faut alors analyser, ce sont donc les mobilisations des acteurs pour problématiser le dispositif, le définir, le configurer et l'intégrer dans leurs propres pratiques ou, au contraire, le délaisser.

La sociologie de la traduction, compte tenu de ses présupposés épistémologiques et théoriques 
ainsi que de sa boîte à outils conceptuelle, permet précisément d'aborder la vidéosurveillance comme un acteur-réseau qui attache ensemble des éléments hétérogènes et qui peut se consolider ou se déliter. Adopter ce prisme théorique est une façon de prendre ses distances vis-à-vis d'analyses qui tendent à isoler le technique du social, en survalorisant les effets de détermination que les technologies pourraient imprimer à l'action et/ou en faisant de la diffusion d'une technologie le résultat de causes extérieures à celle-ci, c'est-à-dire indépendante de ses usages. Ces deux dimensions sont très présentes dans les surveillance studies. D'un côté, ces travaux prennent pour acquis le fait que les dispositifs permettent un contrôle généralisé, ce qui est loin d'être démontré ; d'un autre côté, ils voient dans le développement de la vidéosurveillance un projet global de surveillance de la société. Ces études concluent ainsi pour bon nombre d'entre elles à l'émergence d'une société de surveillance, principalement orchestrée par les États afin de renforcer leur capacité de gouvernement des conduites ${ }^{6}$. Or, il apparaît nécessaire de considérer la vidéosurveillance à travers ses usages réels, sans méconnaître pour autant ce que les caractéristiques techniques de l'outil permettent ou pas de faire et la manière dont par conséquent elles orientent l'action.

Nous avons pris le parti de nous intéresser ici au dispositif sociotechnique en regardant en quoi il peut être, ou non, le support sur lequel se forme un accord entre les acteurs susceptibles de dessiner une action collective. Bien que partiel, l'éclairage ainsi fourni sur les processus de développement de la vidéosurveillance n'en est pas moins substantiel, en montrant comment la vidéosurveillance peut «prendre» ou «ne pas prendre». La décentralisation et l'investissement par les municipalités de la thématique de la sécurité ${ }^{7}$, les logiques de la compétition politique (au niveau local comme au niveau national), les effets de mimétisme d'une ville à l'autre, sont autant d'éléments qui comptent; cependant nous ne les prendrons en compte que dans la mesure où ils sont investis comme des ressources ou des contraintes par les acteurs qui tentent d'en rallier d'autres à la cause de la vidéosurveillance.

Une telle approche suppose une étude empirique fine des usages de la vidéosurveillance, dans une perspective diachronique. Rares sont les recherches qui s'inscrivent dans cette double dimension. Les surveillance studies, déjà évoquées, sont ainsi souvent critiquées pour la rareté ou la fugacité des éléments empiriques sur lesquelles elles reposent ${ }^{8}$. En dehors des nombreuses publications inscrites dans cette veine, les études sur la vidéosurveillance portent principalement sur la mesure de son efficacité ${ }^{9}$ ou, dans le cas français, sur l'analyse du cadre juridique ${ }^{10}$. Certains articles se sont certes intéressés aux usages de la vidéosurveillance pour analyser les régulations induites ${ }^{11}$ ou les effets sur les univers de travail ${ }^{12}$, mais sans que le processus de développement des dispositifs ne soit vraiment scruté ${ }^{13}$.

Pour saisir les mécanismes et circonstances pratiques de genèse et de banalisation du recours à la vidéosurveillance de voie publique, nous avons étudié trois cas d'implantation, à Lyon, Grenoble et Saint-Étienne ${ }^{14}$. L'intérêt de l'étude parallèle de ces trois villes de la région RhôneAlpes est que la place de la vidéosurveillance y 
est inégale : Lyon est la ville qui a le plus, et le plus vite, développé la vidéosurveillance ; à SaintÉtienne, la montée en charge a été un peu plus lente mais la ville dispose aujourd'hui d'un réseau de caméras relativement étendu ${ }^{15}$; à Grenoble enfin, la vidéosurveillance de voie publique a jusqu'à présent été peu déployée, même si des installations localisées ont été faites et si de nouveaux projets ont été lancés à partir de 2008. Les cas étudiés permettent donc de s'interroger à la fois sur les conditions de lancement d'une politique de vidéosurveillance, sur les rythmes différenciés de développement des dispositifs, sur les processus qui font d'une initiative localisée le point de départ d'une politique d'équipement de grande ampleur ou, au contraire, un point d'arrêt, au moins momentané.

Au regard de ces cas d'études, il apparaît que le processus d'intéressement qui sous-tend la formation d'un acteur-réseau de la vidéosurveillance comporte deux dimensions principales : d'une part l'enrôlement ${ }^{16}$ de nouveaux acteurs, au-delà des promoteurs initiaux (1), d'autre part l'invention de nouveaux usages, fondements de nouveaux discours de légitimation (2). C'est par ce double processus qu'est modelée la vidéosurveillance de voie publique dans une ville donnée. Il ne faut évidemment pas y voir deux temps du processus de diffusion mais bien deux dimensions analytiques, largement interdépendantes.

\section{Des promoteurs de la vidéosurveillance à l'enrôlement de nouveaux acteurs}

La diffusion de la vidéosurveillance dans les villes françaises s'appuie sur des promoteurs de la technologie. Au-delà des entreprises qui fournissent l'équipement et des «conseils en sécurité» qui présentent la vidéosurveillance comme un outil majeur des politiques de sécurité, la présence de promoteurs locaux semble essentielle dans le lancement d'un projet d'équipement (1). Cependant, ces promoteurs initiaux ne peuvent être à eux seuls le moteur de l'installation durable du dispositif ; les acteurs chargés de l'opérationnalisation apparaissent déterminants. Leur enrôlement suppose qu'ils parviennent à s'approprier la vidéosurveillance et qu'ils y trouvent motivations ou avantages (2). Mais ce processus d'appropriation est en partie dépendant de la conception et des caractéristiques (techniques et organisationnelles) qui sont imprimées au dispositif ; il peut arriver que l'intéressement échoue et que la consolidation de l'acteur-réseau soit compromise (3).

\section{Le rôle central des promoteurs locaux de la technologie}

Contrairement à la Grande-Bretagne, où le développement de la vidéosurveillance est largement imputable à la politique volontariste du ministère de l'Intérieur ${ }^{17}$, en France ce sont des initiatives municipales qui sont à l'origine des premiers dispositifs de vidéosurveillance de voie publique. C'est notamment M. Balkany qui lance le débat à la fin des années 1980, lorsqu'il annonce son projet d'avoir recours à la vidéosurveillance à Levallois-Perret pour s'attaquer à la délinquance. En 1996, l'installation de caméras par la municipalité de Vaulx-en-Velin relance la controverse. Le positionnement du maire, M. Charrier, ancien membre du PCF qui se revendique de la « gauche alternative », interroge en effet les clivages 
politiques sur les enjeux de sécurité.

De fait, la vidéosurveillance apparaît comme le produit de configurations locales, sans qu'il soit possible de dégager des déterminants simples (couleur politique de la municipalité, niveau de délinquance constatée...). Le recours à la vidéosurveillance est lié à la présence de promoteurs locaux de l'outil, dont le travail porte ses fruits dans des circonstances locales particulières : à l'occasion d'un événement ou d'un projet particulier, qui ouvre en quelque sorte une fenêtre d'opportunité, l'installation de caméras de vidéosurveillance est défendue par un ou plusieurs acteurs municipaux, qui ont en général œuvré à la promotion de l'objet en amont.

Dans les trois villes étudiées, les acteurs et les événements à l'origine de l'installation de caméras de surveillance pour lutter contre la délinquance sont assez différents. À Lyon, avant la première décision municipale d'installer des caméras, la solution est défendue pendant plusieurs années par les commerçants du centre-ville, notamment via des associations locales et leur fédération « Les vitrines de Lyon Presqu'île ». Lorsqu'en 1998 la municipalité cherche une réponse à un phénomène de délinquance particulier - l'attaque de boutiques de luxe par des voitures béliers - la vidéosurveillance est introduite d'autant plus facilement qu'elle est discutée depuis plusieurs années. À Saint-Étienne, après les élections de 1995, l'élu adjoint à la sécurité se fait le porteparole de la vidéosurveillance auprès de l'équipe municipale. Ancien commandant de CRS, il a découvert la vidéosurveillance lors de son passage au Service de sécurité du ministère de l'Intérieur (SSMI). Membre de la majorité mais quelque peu marginal en son sein, il ne parviendra à la faire accepter que lorsque, à l'approche des élections de 2001, la majorité municipale fera de la sécurité une nouvelle priorité. À Grenoble enfin, après la réorganisation des services et l'affichage d'une dimension «sécurité » dans une ville traditionnellement tournée vers la prévention sociale, une ancienne commissaire de police est recrutée à la tête du service " prévention/sécurité » au début des années 2000 ; c'est elle qui introduit l'idée de la vidéosurveillance. Faute de réel soutien de la part des élus, ses initiatives en la matière resteront localisées et prendront notamment la forme d'une expérimentation, lancée en 2004. Avec le changement de composition de la majorité municipale $^{18}$, en 2008 , l'idée d'un équipement de la ville en caméras est inscrite à l'agenda municipal. La construction d'un stade d'agglomération, achevé en 2006, a entre-temps été à l'origine d'une première installation d'ampleur ${ }^{19}$.

Dans les trois villes, ce sont donc des acteurs locaux qui introduisent l'idée d'un recours à la vidéosurveillance ; par ailleurs, même si la promotion de l'outil peut s'appuyer sur des expériences extérieures à la municipalité, c'est bien un événement local (violences, élections municipales, changement de composition de la majorité, construction d'un stade) qui amène le soutien de l'équipe municipale en place et qui permet la concrétisation de l'idée au-delà de l'expérimentation. L'installation d'un système de vidéosurveillance de voie publique peut ainsi être lue comme un choix politique après une période de promotion de l'outil par quelques acteurs, et c'est moins en fonction de l'appartenance partisane des majorités municipales que des configurations politiques locales qu'il faut 
lire ce « déblocage » politique.

\section{Différents mécanismes d'appropriation}

Pour que la vidéosurveillance devienne un outil durable des politiques municipales de sécurité, elle doit intéresser un nombre suffisant d'acteurs, au-delà du cercle des promoteurs initiaux. De fait, l'usage de la technologie est susceptible d'enrôler un certain nombre d'acteurs, pour qui la vidéosurveillance apparaît comme une ressource, matérielle ou symbolique. Les exemples lyonnais et stéphanois permettent de mettre en évidence trois mécanismes d'appropriation de la vidéosurveillance qui consolident l'acteurréseau.

Un premier mécanisme relève de la spécialisation et de la professionnalisation et est lié à la mise en place de services spécifiques en charge de la gestion de la vidéosurveillance et/ou du visionnage des images. Dans la mesure où la vidéosurveillance est la raison d'être de ces services, les professionnels qui y travaillent deviennent des alliés du développement du système. Un tel mécanisme est particulièrement visible à Lyon. Dans cette ville, a été créé en février 2001 un Centre de Supervision Urbaine qui assure sept jours sur sept et toute l'année la gestion des équipements de vidéosurveillance. Composé de seize agents municipaux à l'origine, près de trente en 2007 , le service s'est professionnalisé ${ }^{20}$ et autonomisé ${ }^{21}$. Dans le cas stéphanois, la spécialisation et la professionnalisation des agents en charge de la vidéosurveillance sont beaucoup moins poussées : en effet, les images des caméras installées sur la voie publique sont transférées au centre d'opérations de la police municipale et non à un service spécifique. Les personnes affectées au visionnage sont des agents civils, qui n'ont pas reçu de formation spécifique ${ }^{22}$ et ce sont des policiers municipaux qui assurent la supervision du service ainsi que l'interface avec l'extérieur.

Le cas stéphanois permet en revanche de mettre en avant un deuxième mécanisme d'intéressement, qui repose sur les gratifications symboliques. La vidéosurveillance offre aux policiers municipaux la possibilité de se rapprocher un peu du travail de la police judiciaire, en leur permettant d'intégrer une dimension «investigation» à leur activité. En effet, il arrive que soient réalisées des investigations dans le cadre d'une enquête, à partir des images enregistrées, voire par un visionnage en direct. Si la demande est formulée par la police nationale, le travail est effectué par des policiers municipaux. Comme le souligne l'un de nos interlocuteurs au sein de la police municipale, cette activité suscite un certain intérêt : «Planquer ici pendant 8 jours, c'était super intéressant, tout le monde était content» (entretien 04.07.07). Ce type d'usage, et plus généralement le développement du dispositif, est ainsi encouragé par les gratifications symboliques qu'en reçoivent les policiers municipaux.

Le troisième mécanisme d'appropriation repose sur la constitution de la vidéosurveillance en «ressource à moindre coût» et concerne en particulier la police nationale. À Lyon et Saint-Étienne, la police nationale n'a pas eu à débourser un centime pour l'installation des caméras et des écrans de contrôle mais elle peut avoir la main sur le dispositif : la vidéosurveillance constitue ainsi une aide à moindre coût, aussi bien pour son travail de repérage de faits délictueux (flagrants délits) 
et d'investigation judiciaire (observation en direct ou visionnage d'images a posteriori) que pour ses activités de maintien de l'ordre. La police nationale reste par ailleurs toujours maître de la réponse qu'elle donne aux faits signalés par les centres de visionnage municipaux et, lorsqu'il y a un problème dans un endroit précis, suivant sa propre appréciation, elle peut solliciter des images. Les caméras peuvent aussi aider à calibrer la réponse à apporter suite au signalement d'un fait. La vidéosurveillance apparaît donc comme un « outil en plus », générant peu de contraintes, ce qui explique qu'à Lyon comme à Saint-Étienne la police nationale se dise très favorable au développement de la vidéosurveillance.

\section{Défaut d'intéressement et échec de l'acteur- réseau}

L'intéressement de nouveaux acteurs n'est pas toujours réussi. C'est ce que montre le cas grenoblois : si l'expérimentation lancée en 2004 dans un des quartiers de la ville est restée sans suite, c'est en grande partie parce que les agents en charge de l'opérationnalisation n'ont pas pu s'approprier l'objet. Pour comprendre ce cas, il faut d'abord souligner que, contrairement aux cas lyonnais et stéphanois, aucun agent municipal ne participe à la gestion du dispositif. En effet, faute de soutien politique, il est convenu que l'expérimentation ne doit rien coûter à la municipalité : les promoteurs de la vidéosurveillance (la responsable du service prévention/sécurité de la mairie et le $\operatorname{DDSP}^{23}$ pour l'essentiel) s'appuient donc sur un dispositif national pour le financement ${ }^{24}$ et aucun agent municipal n'est affecté au dispositif. Le monde que les promoteurs de la vidéosurveillance tentent de construire en associant d'autres acteurs ne tient pas ; ce qui est à l'origine de l'échec du dispositif, c'est son incapacité à intéresser les acteurs chargés de le faire fonctionner. Les choix techniques et organisationnels, expression de compromis entre les différents intérêts et positionnements qui se construisent au moment de la mise en œuvre, ont en effet raison du dispositif. La municipalité refusant de mettre des moyens pour la gestion de la vidéosurveillance, l'exploitation des images est confiée à l'hôtel de police de Grenoble. Par ailleurs, du fait des oppositions internes à la majorité municipale vis-à-vis d'une politique d'envergure, l'installation conçue en 2004 est réduite non seulement à une zone précise $\mathrm{e}^{25}$ mais aussi à un objectif limité : le vol à la roulotte dans les voitures. La logique de compression de coûts combinée à celle du repérage de flagrants délits conduit à la décision de faire appel à un « logiciel intelligent », proposé par une entreprise locale, qui permet de faire l'économie de postes d'opérateurs pour l'exploitation en direct des images : les écrans sont en veille et leur allumage est déclenché par le logiciel lorsqu'il détecte une personne restée trop longtemps à proximité d'un véhicule. Cependant, la société informatique locale qui est contactée n'a pas la culture de la sécurité publique ; l'ajustement du logiciel sur la réalité des pratiques de vols à la roulotte est donc difficile et le calibrage en décalage avec ce que les utilisateurs pourraient en attendre. Soit les images arrivent en différé, ce qui ne permet pas l'intervention policière dans une logique de flagrant délit, soit les écrans s'allument trop peu (quand le délai de déclenchement fixé est trop important) ou trop souvent (lorsque le délai fixé est trop court). Par ailleurs, ils ne peuvent pas prendre la main sur les caméras, ils sont donc 
dépendants du cadrage initial, sans possibilité de suivre l'individu qui sort du cadre. Ainsi, les agents de la police nationale censés exploiter les images ne sont pas solidaires de l'innovation parce qu'ils n'en perçoivent pas l'intérêt pour leur travail. Le dispositif devient même une charge quand il se déclenche inutilement, ce qui perturbe leur travail, d'autant que les pannes sont fréquentes. Pour les agents du centre, qui doivent en outre gérer les appels du 17, la vidéosurveillance n'apparaît pas comme un « outil en plus » comme à Lyon ou à SaintÉtienne mais comme « une tâche en plus qui s'est ajoutée » (entretien Police nationale Grenoble, 16.10.2006). Ils se positionnent donc davantage en critiques qu'en alliés de l'innovation et ignorent progressivement l'objet, qui se résume rapidement à quelques écrans morts.

Ce que montrent les trois cas étudiés, c'est que la consolidation de «l'acteur-réseau vidéosurveillance» passe par les organisations gestionnaires de l'équipement, à condition que le dispositif soit mis en place de telle sorte qu'elles puissent en tirer des ressources. Dans certains cas, la mise en œuvre permet de gagner des appuis à travers les usages qu'elle autorise ; dans d'autres cas, l'ajustement entre les préférences des différents acteurs impliqués ne parvient pas à se faire, ce qui produit plutôt des effets de démobilisation et de délitement de l'acteur-réseau. Dans tous les cas, l'enrôlement de nouveaux acteurs va de pair avec l'invention d'usages.

\section{L'invention de nouveaux usages}

La stabilisation d'un système d'action autour de la vidéosurveillance de voie publique ne sup- pose pas seulement l'enrôlement d'un certain nombre d'acteurs. Elle suppose aussi de pouvoir justifier publiquement le dispositif. La capacité des promoteurs de la vidéosurveillance à rendre l'outil légitime apparaît en effet cruciale : cette contrainte concerne en particulier les élus municipaux, vis-à-vis de l'opposition et des électeurs. Or, la vidéosurveillance suscite des controverses assez marquées, qui fragilisent les arguments les plus souvent avancés pour justifier la vidéosurveillance (1). Dans ce contexte, la diversification des usages permet de soutenir de nouveaux arguments de légitimation (2). La conversion observée de certains opposants à la vidéosurveillance municipale soulève alors la question de l'impact de ces modes de légitimation et, plus généralement, celle des ressorts de l'enrôlement des opposants à mesure que le dispositif se développe (3).

\section{L'effet dissuasif : un argument central mais faible}

Les projets municipaux d'installation de vidéosurveillance de voie publique suscitent des oppositions sur deux registres. Un premier registre d'opposition renvoie aux libertés publiques : la vidéosurveillance est critiquée comme attentatoire à des principes fondamentaux comme le droit au respect de la vie privée, la liberté d'aller et venir, le droit à l'image... Ce type d'argument est mis en avant par des organisations de la société civile (le collectif «Non à Big Brother» à Lyon) mais aussi par des élus municipaux. Face à ces critiques, différents aménagements des dispositifs sont réalisés (cf. infra). Face au deuxième grand argument d'opposition, qui relève de l'efficacité ou de l'efficience de l'action publique, les promoteurs de la vidéosurveillance 
sont quelque peu embarrassés : il apparaît en effet difficile de démontrer les effets dissuasifs de la vidéosurveillance, tandis que son coût est lui aisément calculable. Or, cette mise en regard des résultats de la vidéosurveillance et de son coût est régulièrement faite, en particulier par l'opposition municipale. L'argument a d'autant plus de portée qu'il prend place dans un contexte de rationalisation budgétaire.

L'installation de caméras est présentée par ses promoteurs comme devant réduire le nombre de crimes et délits commis sur la voie publique en les rendant plus risqués. La limite de cet argumentaire tient à la difficulté à démontrer l'existence de tels effets : alors que la plupart des études réalisées sur le sujet mettent en avant les problèmes méthodologiques et soulignent la faible plus-value de la vidéosurveillance de voie publique en matière de réduction de la délinquance ${ }^{26}$, l'argument de l'efficacité apparaît difficile à manipuler dans les villes étudiées. À Saint-Étienne, aucun chiffre significatif n'est disponible, faute d'étude évaluative comparant les zones vidéosurveillées à des zones comparables non dotées de caméras ${ }^{27}$. À Lyon, la ville a fait réaliser une analyse portant sur les effets de la vidéosurveillance sur la délinquance de rue mais, face à des conclusions peu encourageantes, elle a choisi de ne pas rendre publique cette étude. En fait, si la plupart des promoteurs de la vidéosurveillance disent croire dans les vertus dissuasives de la vidéosurveillance, ils ont souvent peu de données pour étayer leurs dires.

Face aux arguments des opposants, la défense de la portée dissuasive de la vidéosurveillance a donc peu d'effet. Il est frappant de voir que d'autres arguments prennent rapidement le dessus. L'invention de nouveaux usages apparaît alors déterminante dans l'ancrage de la vidéosurveillance car elle fournit de nouveaux modes de légitimation.

\section{La diversification des usages comme support de légitimité}

Les usages de la vidéosurveillance sont largement inventés chemin faisant : l'inscription d'une technologie dans un système social crée des opportunités d'usages. Développement de nouvelles pratiques et déploiement de nouveaux arguments de légitimation se nourrissent alors l'un l'autre, comme le montre l'importance prise par trois grands types d'usages dans le discours des promoteurs de la vidéosurveillance dans les villes étudiées : l'identification d'auteurs de crimes et délits, le calibrage des réponses policières et la gestion des petits désordres. Ces usages permettent de légitimer la vidéosurveillance comme instrument de répression d'une part, comme aide logistique d'autre part.

Un des éléments marquants est l'orientation vers le repérage de faits délictueux et l'interpellation de leurs auteurs. La conception même des dispositifs est révélatrice de l'intégration de cette dimension. Dans les trois villes étudiées, aucun effort particulier n'est fait sur la signalétique informant sur la présence de caméras, ce qui limite l'impact dissuasif. Les systèmes mis en place à Lyon et à Saint-Étienne comportent des centres de visionnage en continu pour repérer des comportements suspects ou des délits et avertir immédiatement la Police nationale ou municipale. Par ailleurs, à Saint-Étienne, le décompte des délits observés 
caméra par caméra permet de repérer des caméras 'inutiles'; leur déplacement sera alors encouragé par les services de police ${ }^{28}$. Dans une logique dissuasive, le fait que ces caméras ne rapportent rien serait au contraire vu comme une preuve de leur efficacité. La construction des dispositifs semble donc répondre à une logique policière et judiciaire de traitement des crimes et délits, plus qu'à une logique de prévention ou de dissuasion. Ceci peut s'expliquer par la forte présence des acteurs policiers dans les réseaux de promotion de la vidéosurveillance, les acteurs municipaux les plus actifs dans ce domaine étant souvent d'anciens policiers. Ce sont d'ailleurs également les policiers qui développent un autre type d'usage : le visionnage a posteriori des images filmées par les caméras, dans le cadre d'une enquête judiciaire. Ces usages répressifs sont aisément quantifiables, à la différence des effets dissuasifs. Par ailleurs, ils peuvent être « (dé)montrés » par des faits particulièrement marquants. À Lyon comme à Saint-Étienne nous avons pu observer la construction d'un discours de légitimation de la vidéosurveillance qui s'appuie à la fois sur des statistiques de faits repérés et sur la circulation de « petites histoires » prenant la forme de récits emblématiques, systématiquement repris pour illustrer l'intérêt du dispositif ${ }^{29}$. Pour ce qui est des statistiques, le CSU de Lyon comme la police municipale de Saint-Étienne tiennent des comptes précis des faits repérés grâce aux caméras et des suites qui leur sont données. Bien que ces chiffres soient faibles au regard du nombre de crimes et délits, les autorités municipales en font un argument pour montrer que l'outil sert à quelque chose.

Dans la mesure où les dispositifs de vidéosur- veillance, d'initiative municipale, prévoient des liaisons (y compris vidéo) avec la police nationale dans l'optique de lui signaler des faits, cette dernière utilise aussi la vidéosurveillance pour mieux gérer ses interventions. En effet, les écrans dont la police nationale dispose dans ses locaux lui permettent d'avoir directement accès aux images, qui sont alors une aide logistique, pour les interpellations, les opérations de maintien de l'ordre ou encore le suivi de manifestations : «C'est d'un très gros intérêt pour nous également en matière d'ordre public, pour le suivi des manifestations. (...) Ça permet d'optimiser notre boulot, de ne pas mettre des gens en danger, de créer le moins de gêne possible à la population. (...) Pourquoi envoyer quatre véhicules avec quatre fonctionnaires à l'intérieur sur un différend qui oppose deux poivrots? Mais les deux poivrots quand vous êtes aveugles dans la salle de commandement, ils peuvent vous être annoncés comme une rixe phénoménale. " (entretien police nationale Saint-Etienne, 09.10.06). Un tel usage de la vidéosurveillance, se retrouve au sein des polices municipales : pour vérifier qu'un problème signalé (un stationnement gênant par exemple) est réel avant de mobiliser des agents, pour vérifier qu'un contrôle routier se passe bien ${ }^{30} \ldots$

L'intérêt de la vidéosurveillance comme «aide logistique» est également mis en avant pour des usages autres que ceux liés à la lutte contre la délinquance : la vidéosurveillance est présentée comme un outil de lutte contre les petits désordres urbains. Au départ très focalisé sur la sécurité au sens de lutte contre la délinquance de rue ${ }^{31}$, le CSU de Lyon s'assigne aujourd'hui une fonction large, comme l'explique son responsable : « Ça peut faire sourire certains mais on essaie aussi 
de montrer que la vidéosurveillance peut être utile à d'autres fins que la protection, la sécurité immédiate. On est dans la gestion urbaine de proximité. On n'oublie pas nos fonctions premières mais on exploite au maximum le dispositif. (...) La vidéosurveillance peut avoir un regard très neutre et très complet de la voie publique et permettre y compris le signalement de SDF en difficulté à la veille sociale, avec laquelle le CSUL a un partenariat " (entretien 04.04.07). A Saint-Étienne également, ce type d'usage revient souvent dans le discours des acteurs, qu'il s'agisse de la lutte contre les incendies ou les inondations ou l'aide à la manœuvre d'un camion coincé dans une petite rue.

La vidéosurveillance de voie publique devient ainsi en quelque sorte la «bonne à tout faire» de la gestion urbaine. Aux usages évoqués s'ajoute d'ailleurs l'évocation de la lutte contre le terrorisme, l'exemple britannique faisant office d'argument ${ }^{32}$. Cette pluralité d'arguments de légitimation, liée à une diversification des usages, permet à chaque acteur associé au dispositif de développer une logique propre de justification tandis que, pour les élus municipaux, elle permet de sortir d'une approche de l'efficacité qui se focaliserait uniquement sur la diminution des crimes et délits : c'est finalement sa multifonctionnalité qui peut légitimer la vidéosurveillance.

\section{Développement du dispositif sociotechnique et conversion des opposants}

La banalisation de la vidéosurveillance dans les villes françaises relève aussi d'un affaiblissement des oppositions. De fait, à Lyon comme à SaintÉtienne, le positionnement de certains élus a évo- lué, au point de voir les anciens opposants devenir des alliés. À Lyon, au moment des premières décisions, en 1999/2000, la majorité RPR/UDF/ Milloniste ne rencontre que l'opposition affirmée des Verts, qui sont les seuls élus à voter contre, tandis que quelques autres élus (GAEC, RG, $\mathrm{PC}^{33}$ ) s'abstiennent en insistant sur le nécessaire respect des libertés publiques. Après les élections municipales de 2001 et l'accession au pouvoir d'une majorité de gauche plurielle, les Verts, qui font désormais partie de la majorité, continuent à voter contre, les élus GAEC jouant le jeu de la majorité et soutenant les décisions d'installation de caméras. Cependant, à partir de décembre 2005, certaines décisions d'extension reçoivent le soutien des élus Verts, tandis qu'ils s'abstiennent sur d'autres propositions. L'opposition n'est donc plus de principe mais de circonstance. Les oppositions s'affaiblissent aussi à Saint-Étienne, de manière plus nette. En septembre 2000, les élus d'opposition PS, PC et Verts votent contre la première décision municipale relative à l'installation de caméras. Après les élections de 2001, qui reconduisent une majorité UDF-UMP ${ }^{34}$, les élus PS changent de positionnement et approuvent de nouvelles installations, position qu'ils ont gardée depuis. Les élus Verts et PC votent contre l'installation de nouvelles caméras en 2002 mais les Verts votent favorablement ou s'abstiennent à partir de 2003, seuls les trois élus communistes ou apparentés continuant à voter contre. C'est l'évolution des socialistes qui est ici la plus notable, car elle a lieu dès 2002. Ainsi, ce n'est pas leur accession à la municipalité en 2008 qui fait évoluer leur discours, ni même l'approche de ces élections.

Pour expliquer ces changements de position, 
on peut avancer l'hypothèse que c'est le développement même du dispositif qui conduit à la conversion des opposants en alliés, soit parce que leurs arguments en sont affaiblis, soit parce que le mode de développement de la vidéosurveillance entraîne les opposants dans le dispositif. L'évolution de l'opposition stéphanoise semble relever du premier cas de figure. En 2000, l'opposition de gauche motive son vote contre la vidéosurveillance en insistant sur les dangers pour les libertés individuelles et en mettant en doute l'efficacité du dispositif; en 2002, les élus du parti socialiste se disent rassurés par le fonctionnement du dispositif pour ce qui est des libertés publiques mais aussi de l'efficacité du système : «Le procureur notamment m'a rassuré sur les précautions prises en matière de libertés dans le cadre de la loi du 21 janvier 1995. J'ai noté que la commission présidée par un magistrat a joué son rôle et que les images sont détruites dans les délais prévus et que les précautions sont prises s'agissant des caméras qui risqueraient de s'orienter vers la vie privée des personnes, dans les appartements notamment. Il y a bien des obturations telles qu'elles ont été prévues par la loi. Par ailleurs, l'implantation que vous nous proposez ne se limite plus à l'hyper centre et s'inscrit dans une meilleure cohérence. " (intervention élu socialiste en conseil municipal, 07.01.02). Il resterait cependant à démontrer que les élus socialistes ont vraiment été rassurés par le fonctionnement du dispositif et que l'évolution de leur positionnement ne s'explique pas par des formes d'échanges politiques.

Dans le cas lyonnais, le processus à l'œuvre est d'une certaine façon plus lisible et paraît plus directement lié au mode de développement du dispositif sociotechnique. D'un côté, c'est la poursuite du développement de la vidéosurveillance par une nouvelle majorité, qui conduit certains élus à changer de positionnement : les élus du GAEC, membres de la nouvelle majorité, passent de l'abstention au soutien pour marquer leur alliance. Quant aux Verts, s'ils ne jouent pas la solidarité au sein de la «gauche plurielle» et maintiennent leur opposition, ils sont progressivement enrôlés, notamment via le collège d'éthique $^{35}$ installé en 2004. Les élus Verts refusent de prendre part au vote sur la création du collège et sont très critiques sur l'initiative. Cependant, une fois le comité installé, ils demandent à en faire partie, pour mieux contrôler le développement de la vidéosurveillance : "Est ce que la vidéosurveillance ne doit être faite que par des gens qui sont pour? Est ce que justement il ne faut pas des gens qui soient des contre vidéo, pour qu'elle soit faite avec le plus d'éthique possible » (entretien adjoint Vert à la ville de Lyon, 13.04.04) ${ }^{36}$. Un élu Vert sera effectivement intégré dans le comité, qui fait ainsi entrer les opposants dans l'acteurréseau. Dans ces conditions, il est moins étonnant de voir les élus Verts voter pour certains projets d'extension à partir de 2005.

Usages et encadrement des usages participent ainsi de la consolidation de l'acteur-réseau dans les villes de Lyon et de Saint-Étienne, en fournissant de nouveaux arguments de légitimation et en enrôlant non seulement les usagers de la technologie mais aussi certains opposants.

\section{Conclusion}

L'analyse de l'équipement en vidéosurveillance 
de voie publique des villes de Lyon, Saint-Étienne et Grenoble montre, en plein ou en creux, que son développement découle de l'enrôlement progressif d'acteurs professionnels et politiques, qui inventent, investissent et/ou légitiment divers usages. En cela, la vidéosurveillance s'apparente bien à un dispositif sociotechnique : ni les acteurs partie prenante du système d'action ni les usages ne sont prédéterminés.

Au-delà des enseignements empiriques sur le développement de la vidéosurveillance en France, l'analyse développée permet de souligner les apports et les limites d'une approche de l'action publique qui s'appuie sur les concepts de la sociologie de la traduction. Un des intérêts de l'approche par l'acteur-réseau est d'inviter à prendre au sérieux les «échecs» comme les «réussites» et à les analyser de la même façon. L'étude parallèle des cas grenoblois, stéphanois et lyonnais montre ainsi que ce qui est déterminant est moins la présence d'un certain nombre de paramètres (couleur politique de la municipalité, taux de criminalité...) que la forme que prend le dispositif, en fonction des configurations qui le portent : les modalités de l'expérimentation grenobloise ne parviennent pas à faire entrer de nouveaux alliés dans l'acteur-réseau. Le deuxième enseignement est que le processus de production du dispositif sociotechnique conditionne la forme que prend l'objet technologique : l'enrôlement des acteurs policiers favorise l'usage de la vidéosurveillance à des fins d'identification et pousse à l'amélioration et à la sophistication des capacités techniques des équipements.

Si les processus mis en évidence éclairent l'extension de la vidéosurveillance dans les villes françaises au cours des dernières années, l'effet des évolutions récentes devra être interrogé. Le soutien national apporté depuis 2007 aux projets municipaux ${ }^{37}$ ne constitue finalement qu'une ressource supplémentaire à disposition des défenseurs locaux de la vidéosurveillance ; cependant, la pression du gouvernement se fait plus pressante, comme l'illustre la possibilité évoquée dans le cadre de la LOPPSI $2^{38}$ en cours de discussion de permettre à l'État d'imposer des caméras aux communes. 


\section{$R \cdot E ́ \cdot F \cdot E \cdot R \cdot E \cdot N \cdot C \cdot E \cdot S$}

M. AKRICH, « La construction d'un système socio-technique », in M. Akrich, M. Callon et B. Latour, Sociologie de la traduction, Paris, Presses de l'Ecole des Mines, 2006, pp.109-134.

M. BEETSCHEN, Vidéosurveillance et processus décisionnel : l'exemple lyonnais, mémoire IEP Grenoble, 2004, 123 p.

D. BOULLIER, « La vidéosurveillance à la RATP : un maillon controversé de la chaîne de production de sécurité », Les Cahiers de la sécurité intérieure, n²1, 1995, pp. 88-100.

M. CUSSON, La surveillance et la télésurveillance : sont-elles efficaces ?, Revue Internationale de Criminologie et de Police Technique et Scientifique, vol. LVIII, 2005, pp.131-150.

L. Dumoulin, S. GERMAin, A.-C. DOUILLET, «Une petite entreprise qui ne connaît pas la crise. Le succès de la vidéosurveillance au regard de la littérature internationale », Champ pénal, 2010.

J.-C. FROMENT, « Regard juridique sur la vidéosurveillance urbaine : un droit en trompel'œil », La semaine juridique, Edition Adm. et collec. Terr., n¹3, 27 mars 2006, pp.435-440.

E. HEILMANN et M.-N. MORNET, « L'impact de la vidéosurveillance sur les désordres urbains, le cas de la Grande-Bretagne », Les Cahiers de la Sécurité Intérieure, vol.46, n4, 2001, pp.197-211. L. HEMPEL et E. TÖPFER, CCTV in Europe. Final Report, Berlin, Centre for Technology and Society, 2004.

T. LE GOFF, « L'insécurité 'saisie' par les maires », Revue française de science politique, vol.55, n³, 2005, pp.415-444.

D. LYON, Surveillance Society. Monitoring everyday life, Buckingham, Open University Press, 2001.

G. T. MARX, «'I'll be Watching You': Reflections on the New Surveillance », Dissent, winter 1985, pp.26-34.

D. NEYLAND, Privacy, Surveillance and Public Trust, Palgrave Macmillan, Basingstoke, 2006.

C. NORRIS et M. McCAHILL, «CCTV: beyond penal modernism?», British Journal of Criminology, vol.46, pp.97-118, 2006.

C. NORRIS, J. MORAN et G. ARMSTRONG (dir.), Surveillance, CCTV and social control, Burlington, Ashgate, 2006.

F. OCQUETEAU et M.L. POTTIER, «Vidéosurveillance et gestion de l'insécurité dans un centre commercial : les leçons de l'observation », Les cahiers de la sécurité intérieure, $\mathrm{n}^{\circ} 21,1995$, pp.60-74.

M.-C. RENARD, Les enjeux sociaux de la vidéosurveillance. Processus de mise en place d'un nouvel outil sécuritaire dans les espaces publics: l'exemple lyonnais, mémoire de fin d'études, Lyon, ENTPE, 2001.

S. ROCHÉ, A.-C. DOUILLET, L. DUMOULIN, S. GERMAIN, N. CAMERATTI, Les usages techniques de la vidéosurveillance : une comparaison entre Lyon, Saint-Étienne et Grenoble, rapport de recherche, 2007, 299 p.

SÉNAT, Vidéosurveillance : pour un nouvel encadrement juridique, Rapport d'information de MM. Jean-Patrick Courtois et Charles Gautier, fait au nom de la Commission des lois, 10 décembre 2008.

B. WELSH B., D. FARRINGTON, Effects of Closed Circuit Television Surveillance on Crime, Campbell Systematic Reviews, vol. 17, 2008, pp.2-73. 
$\mathrm{N} \cdot \mathrm{O} \cdot \mathrm{T} \cdot \mathrm{E} \cdot \mathrm{S}$

1. Cet article est la version remaniée d'une communication à l'atelier « Les approches pragmatiques de l'action publique », 27-28 mai 2009, Congrès de la Société québécoise de science politique, Ottawa. Nous remercions les organisateurs, Christelle Routelous, Virginie Tournay, Isabelle Vedel et tout particulièrement Fabrizio Cantelli pour ses judicieux commentaires.

2. Dans les lieux publics ou ouverts au public.

3. «Michèle Alliot-Marie : 'Je compte tripler le nombre de caméras de vidéosurveillance d'ici fin 2009'», Le Monde, 13.10.2007.

4. Selon un rapport parlementaire qui s'appuie sur les chiffres du ministère de l'Intérieur, le nombre de communes ayant recours à la vidéosurveillance (y compris pour la surveillance de bâtiments publics ou la régulation du trafic) s'élevait à 1.522 à la fin 2007, contre 1.142 fin 2006 et 812 fin 2005. (Sénat, Vidéosurveillance : pour un nouvel encadrement juridique, Rapport d'information de MM. JeanPatrick Courtois et Charles Gautier, fait au nom de la Commission des lois, 10.12.2008). En 2008, le ministère de l'intérieur estimait à 22.000 le nombre de caméras de voie publique.

5. Voir par exemple : M. Akrich, « La construction d'un système sociotechnique ", in M. Akrich, M. Callon et B. Latour, Sociologie de la traduction, Paris, Presses de l'école des Mines, 2006, pp. 109-134.

6. Voir par exemple : G. T. Marx, "'I'll be Watching You': Reflections on the New Surveillance », Dissent, winter 1985, pp. 26-34 ; D. Lyon, Surveillance Society. Monitoring everyday life, Buckingham, Open University Press, 2001.

7. Tanguy Le Goff, «L'insécurité 'saisie' par les maires », Revue française de science politique, vol.55, n³, 2005, pp. 415-444.

8. Clive Norris et Michael McCahill, «CCTV: beyond penal modernism?», art. cité, p. 98.

9. Pour une synthèse sur ce sujet : M. Cusson, « La surveillance et la télésurveillance sont-elles efficaces? ", Revue Internationale de Criminologie et de Police Technique et Scientifique, vol. LVIII, 2005, 131-150.

10. À titre d'illustration : J.-C. Froment, « Regard juridique sur la vidéosurveillance urbaine : un droit en trompe-l'œil », La semaine juridique, Edition Adm. et collec. Terr., n¹3, 27 mars 2006, pp. 435-440.

11. F. Ocqueteau et M.L. Pottier, «Vidéosurveillance et gestion de l'insécurité dans un centre commercial : les leçons de l'observation », Les cahiers de la sécurité intérieure, $\mathrm{n}^{\circ} 21,1995$, pp. 60-74; D. Boullier, « La vidéosurveillance à la RATP : un maillon controversé de la chaîne de production de sécurité », Les Cahiers de la sécurité intérieure, n²1, 1995, pp. 88-100.

12. D. Neyland, Privacy, Surveillance and Public Trust, Palgrave Macmillan, Basingstoke, 2006.

13. Pour une revue de la littérature sur la vidéosurveillance voir : L. Dumoulin, S. Germain, A.-C. Douillet, « Une petite entreprise qui ne connaît pas la crise. Le succès de la vidéosurveillance au regard de la littérature internationale », Champ pénal, 2010

14. Voir: S. Roché et al. (2007), Les usages techniques de la vidéosurveillance : une comparaison entre Lyon, Saint-Étienne et Grenoble, 2007, 299 p. Le travail a été réalisé en réponse à un appel à projet de l'Institut National des Hautes Etudes sur la Sécurité, intitulé « espaces publics et sécurité » (2005).

15. à Lyon, la première décision d'installation de caméras à fin de « sécurisation » de la voie publique date de 1999 ; il y avait fin 2007 environ 180 caméras (plus 29 autres prévues pour 2008), pour une ville de 400.000 habitants située dans une agglomération de 
1,2 million d'habitants. A Saint-Étienne, la première décision en la matière remonte à 1997 ; il y avait fin 200967 caméras, pour une ville de 180.000 habitants située dans une agglomération de 390.000 habitants. 16. L'enrôlement est un intéressement réussi : le processus d'enrôlement attribue à un rôle à un acteur, lui donne une place au sein de l'acteur réseau.

17. E. Heilmann et M.-N. Mornet, « L'impact de la vidéosurveillance sur les désordres urbains, le cas de la Grande-Bretagne ", Les Cahiers de la Sécurité Intérieure, vol.46, nº 4, 2001, pp. 197-211

18. Après 2008, les Verts ne font plus partie de la majorité municipale.

19. Le bâtiment est équipé de 82 caméras, qui filment de façon assez large la voie publique.

20. Les opérateurs reçoivent une formation de trois semaines, sur les plans éthique, juridique, technique et opérationnel. Par ailleurs le pôle technique du CSUL a développé une forme d'expertise sur les questions de vidéosurveillance. Il est ainsi consulté par la commission communale de prévention situationnelle, chargée de statuer sur les projets d'aménagement sous l'angle de la sécurité.

21. Le pôle administratif et financier du CSUL assure en effet une certaine autonomie, revendiquée par le responsable du service, qui n'est lié hiérarchiquement ni à la police nationale ni à la police municipale (entretien 04.04.07).

22. Au moment de l'enquête (juillet 2007) un projet de formation était en cours de montage.

23. Directeur départemental de la sécurité publique.

24. Le plan «25 quartiers», lancé par le gouvernement en 2004.

25. 3 caméras sont placées dans une rue.

26. Sur les difficultés de ces évaluations et leurs résultats ; B. Welsh B., D. Farrington, Effects of Closed Circuit Television Surveillance on Crime, Campbell Systematic Reviews, vol. 17, 2008, p. 2-73.
27. Et les chiffres globaux ne dessinent pas de tendance claire (taux de criminalité dans la circonscription de sécurité publique de Saint-Étienne : 74,35\%o en 2004 ; 71,96\% en 2005, 73, 42 \% en 2006).

28. Entretien Police municipale Saint-Étienne 04.07.07.

29. Comme le fait qu'à Saint-Etienne le réseau de vidéosurveillance a permis d'interpeller des malfaiteurs chevronnés en plein cambriolage. Rapporté dans le journal municipal (Aujourd'hui Saint-Étienne, 24 février 2006, p.9), ce cas a aussi été évoqué par plusieurs de nos interlocuteurs en entretien.

30. Il s'agit alors de protéger les policiers qui effectuent le contrôle.

31. «La priorité, c’est la sécurité. Le fait de voir une voiture mal stationnée, ça pourrait intéresser la police municipale, mais on sépare complètement ces deux aspects. Ils ne sont pas là pour diriger la police municipale. C'est comme s'ils voyaient un papier sur le trottoir et qu'ils appelaient le service propreté » (Entretien avec le premier responsable du CSUL, cité par M.-C. Renard, Les enjeux sociaux de la vidéosurveillance. Processus de mise en place d'un nouvel outil sécuritaire dans les espaces publics: l'exemple lyonnais, mémoire de fin d'études, Lyon, Ecole nationale des travaux publics de l'Etat, 2001, p.119).

32. «N'empêche que l'Angleterre, lors des événements de Londres, a bien montré que c'était quand même bien utile » (entretien élu, 23.11.06).

33. GAEC : Gauche alternative écologiste et citoyenne ; RG : Radicaux de gauche ; PC : Parti communiste.

34. L'UMP ayant succédé au RPR en 2002.

35. Il s'agit d'une commission extra-municipale présidée par un conseiller d'Etat et composée d'élus, de personnalités qualifiées et de représentants d'associations (18 personnes) : tous les projets 
d'extension lui sont présentés ; il formule des suggestions d'amélioration du dispositif et répond aux réclamations que peuvent faire les habitants.

36. Entretien réalisé par Marion Beetschen pour son mémoire à 1'IEP de Grenoble: Vidéosurveillance et processus décisionnel : l'exemple lyonnais, 2004

37. Selon une circulaire du 21 février 2008, l’État a contribué en 2007 au financement de 309 projets pour un montant de 13,4 millions d'euros, par l'intermédiaire du FIPD (Fonds Interministériel de Prévention de la Délinquance inscrit dans la loi du 5 mars 2007).

38. Loi d'Orientation et de Programmation de la Performance de la Sécurité Intérieure. 